\title{
TATTOO - FORMAL INDICATION OF RADIOISOTOPE USE TO LOCATE SENTINEL LYMPH NODE? REPORT OF TWO CLINICAL CASES
}

Paulus F. M. Ramos¹, Claudia A. A. Góes', Tatiana P. B. B. Figueiredo', Rafael A. M. Hassan¹, Naiana S. C. Andrade', Rodrigo S. C. Guindalini ${ }^{1}$

${ }^{1}$ Clínica CAM/CLION - Salvador (BA), Brazil.

Introduction: Tattoos have gained popularity in recent years and have become socially accepted between men and women. It is estimated that $14 \%$ of the American population has at least one. The process of skin pigmentation creates an inflammatory reaction and in a few days the macrophages encompass the injected pigments. The carbon particles migrate through the lymphatics and within 3 days the pigments can already be seen in the lymph nodes. Tattoo ink and inflammation can block lymphatic chains, influencing detection and sensitivity, causing false-negatives in sentinel lymph node rates. Case-report: Two women aged 46 years, with breast cancer. The first one with left breast breast lesion and submitted to quadrantectomy with sentinel lymph node biopsy. The second had bilateral breast cancer and underwent skin-spared adenomastectomy. Both had a tattoo on the back ipsilateral to the tumor and underwent sentinel lymph node biopsy with the radiopharmaceutical technique alone. The lymph nodes were detected with the radioisotope and a parasitizing lymph node of blackened aspect that did not capture. The pathological anatomical study confirmed the presence of the tattoo pigment in the lymph nodes stained. Discussion: Axillary staging continues to be an important prognostic factor in breast cancer in the last decade, lymphadenectomy has been replaced by the search for sentinel lymph nodes in clinically no axilla without compromising. The combined technique using radioisotope and patent blue results in higher rates of sentinel node identification. The popularization of the skin tattoo may bring a new paradigm for the surgical staging of the armpit. The pigments can drain to the locoregional lymph nodes causing inflammation and consequent obstruction of the lymphatic channels, compromising their biopsy. The pigment may further hide the presence of metastatic cells or promote detection of the false sentinel if used only with the use of patent blue. Due to the pigmentation of regional lymph nodes by tattoo ink, there may be a failure to identify the true sentinel, and a "pseudosentinella" is identified. When they are located superficially in the armpit they can mimic the lymph node dyed blue, causing the true sentinel to be lost and the patient to undergo staging. Conclusion: Breast surgeons should be aware of the presence of tattoos in patients who will do sentinel lymph node screening, especially those near the drainage area to be screened. This may lead to the choice of technique and may be an indication of the use of the radioisotope as a method of choice or at least a combined technique. The use of only the blue patent may confuse the surgeon and remove only the lymph node stained by the tattoo, generating a false negative in the assessment of the axilla and impairing oncologic staging. 